\title{
Paradojas de la evaluación en el contexto de los modelos basados en competencias. La voz de los estudiantes
}

\author{
Gerardo I. Sánchez ${ }^{1}$ y Ximena E. Jara ${ }^{2}$ \\ (1) Centro de Investigación en Educación para la Justicia Social Universidad Católica del Maule (CIEJUS), Talca - Chile. \\ (correo-e: gsanchez@ucm.cl) \\ (2) Universidad Autónoma de Chile, Pedagogía en Historia, Geografía y Ciencias Sociales, Chile \\ (correo-e: xjaraa@autonoma.cl)
}

Recibido Oct. 21, 2020; Aceptado Dic. 21, 2020; Versión final Ene. 22, 2021, Publicado Jun. 2021

\begin{abstract}
Resumen
Este artículo problematiza las principales paradojas que enfrenta la evaluación del aprendizaje en relación a los modelos basados en competencia en el contexto universitario. Mediante una aproximación cualitativa se administran entrevistas semiestructuradas construidas a partir del concepto de paradojas a 35 estudiantes de pedagogía. El análisis permite identificar paradojas asociadas a aprender versus aprobar; proceso versus resultados; desarrollo del pensamiento versus reproducción; y mejorar versus conservar. La práctica evaluativa reflejaría la distancia que muestra la docencia universitaria respecto de los actuales modelos basados en competencias; y las dificultades para articular las funciones de selección y de formación, a partir del ejercicio de la retroalimentación. Se concluye que la evaluación de estudiantes se instrumentaliza en el aula universitaria bajo la lógica de la comprobación de un aprendizaje situado al final del proceso bajo criterios de selección y control que implican el uso punitivo del error.
\end{abstract}

Palabras clave: paradojas en la evaluación; modelos basados en competencia; retroalimentación y aprendizaje

\section{Evaluation paradoxes in the context of competency-based models. Students' voices}

\begin{abstract}
The present study aims to examine learning assessment paradoxes in university competency-based models. Semi-structured interviews are conducted based on the concept of paradoxes and by using a qualitative approach. The sample consists of 35 pedagogy students. The results show paradoxes associated with: learning versus passing, process versus results, thought development versus reproduction, and improving versus maintaining. There is disconnect in student evaluation processes between university teaching and current competency-based models. In addition, there are difficulties in articulating selection and training functions based on feedback exercises. It is concluded that student evaluations are used as tools in university classrooms by applying a logic that involves penalizing errors to verify learning at the end of the learning process.
\end{abstract}

Keywords: evaluation paradoxes; competency models; feedback and learning 


\section{INTRODUCCIÓN}

Chile viene implementando políticas orientadas a mejorar la calidad y equidad de la educación en tanto mecanismo de movilidad social que, en el contexto universitario a involucrado inversión en infraestructura, programas de acceso inclusivo, aumento de financiamiento para familias vulnerables, gratuidad, y por supuesto, cambios curriculares. En el escenario actual se observa, por una parte, la diversificación en los perfiles de quienes ingresan a las universidades, derivado de una creciente masificación y por otra, la instalación de nuevos modelos formativos que aseguren las competencias requeridas en los futuros profesionales. Este trabajo se sitúa en la Universidad Católica del Maule, donde la matrícula de pregrado es de estudiantes preferentemente de los tres primeros quintiles según la distribución de ingresos de sus familias y que en su mayoría no tienen una formación profesional. Desde el año 2015 la Universidad suscribe un modelo educativo orientado a competencias y resultados de aprendizaje, iniciando la implementación sostenida de procesos de innovación y renovación curricular.

En este ámbito, la docencia universitaria se enfrenta a una población masiva y diversa de estudiantes a la cual es preciso proveer de las capacidades requeridas que les permitirán enfrentar el mundo complejo e incierto en el que participarán como profesionales. Una docencia efectiva será aquella capaz de reorientar sus dispositivos didácticos y evaluativos para lograr que la mayor parte de los estudiantes, independientemente de su origen socioeconómico y cultural, desarrolle las competencias necesarias vinculadas al ejercicio de su profesión. Ahora bien, en el marco de una institución en cuya tradición la educación estaba reservada para una élite, y por tanto, concebida a los fines de la homogeneización social y la selección de los estudiantes, "tarde o temprano, evaluar es crear jerarquías de excelencia, en función de las cuales se decidirán el progreso en la trayectoria escolar, la selección para ingresar en la enseñanza secundaria, la orientación hacia distintas modalidades de estudios, la calificación para ingresar al mercado laboral de trabajo y a menudo la obtención efectiva de empleo" (Perrenoud, 2008, p.7). Sin embargo, apremiada por los nuevos desafíos que se le plantean en el presente, la educación superior experimenta la necesidad de superar en sus prácticas el modelo tradicional humboldtiano y su enfoque academicista para transitar a modelos más inclusivos en escenarios de creciente masificación caracteristicos de las sociedades contemporáneas, los que implican un cambo significativo respecto al concepto de universidad selectiva o de élite (Juarros, 2006).

La opción por modelos basados en competencias se ve enfrentada a un conjunto de decisiones de gestión académica y administrativa nada fácil de resolver, dada la diversidad de lógicas de trabajo vigentes al interior de las instituciones terciarias, y que exige la formación del profesorado universitario para la implementación de la evaluación por competencias, junto a una formación con la necesaria pertinencia (lon y Cano, 2012; Jover y García, 2015) y centralidad en el estudiante y su aprendizaje. La educación superior tiene el imperativo social de desarrollar en los estudiantes las competencias profesionales y genéricas que aseguren los desempeños que el medio laboral demanda (Villardón, 2006). Para ello, las Universidades chilenas adscritas a los requerimientos de los procesos de acreditación institucional y de carrera, en su mayoría han suscrito modelos relacionados a competencias con la intención de favorecer procesos de innovación en sus enfoques curriculares que logren alinear virtuosamente enseñanza, aprendizaje y evaluación.

Estos modelos formativos definen como centro del proceso de enseñanza - aprendizaje el quehacer del estudiante, y ello supone un profesorado capaz de ceder el escenario, el protagonismo, la palabra, el tiempo y, en definitiva, el control sobre el aprendizaje a medida que avanza el proceso (Beneitone, et al., 2007). Los planteamientos anteriores son expresión de la profunda discusión que en el último tiempo viene desarrollando la institucionalidad universitaria respecto a cómo innovar las prácticas pedagógicas que ancladas al verbalismo y la exposición, la reproducción, el control y la selección ponen en peligro la posibilidad de asegurar una formación que responda a los retos del siglo XXI (Tejada y Ruiz, 2016; Jover y García, 2015).

Así, los enfoques de competencia en sus diversas expresiones, surgen como respuesta al imperativo de una formación que integre distintos saberes (conceptuales, procedimentales y actitudinales) capaces de ser movilizados en contextos vinculados al ejercicio del desempeño profesional (Chan, et al., 2017). Ello supone la incorporación de nuevas claves en la docencia universitaria, dentro de las cuales figuran por su efecto condicionador la escasa formación docente del profesorado universitario (Parris y Saville, 2011), y la racionalidad que muestran los procesos de evaluación formativa que se logran implementar (Sadler, 2010; Evans, 2013). Como parte de los esfuerzos institucionales por innovar, la evaluación que cumple una función social de certificación de las competencias alcanzadas, y una función de carácter pedagógico, orientada a proveer evidencias que permitan regular el proceso de enseñanza - aprendizaje, está llamada a ocupar un lugar dinamizador en los procesos de diseño y desarrollo curricular.

La evaluación constituye el punto de encuentro del acto didáctico. En la perspectiva de Biggs (2007), ha de asegurar el alineamiento constructivo entre el alumno, el profesor y el contenido, pues, afecta al resto de los elementos, con la intención no solo de verificar su aplicación, implementación y productos, sino, sobre todo, 
con el propósito de mejorarlos. Más específicamente, provee información y genera consecuencias para el docente, el estudiantado y el sistema en su conjunto (Villardón, 2006); condiciona el accionar del estudiante en su proceso de aprendizaje (Murphy, 2010); y, determina la profundidad de que lo se aprende y se considera valioso en el proceso formativo (Struyven, et al., 2005). La constante evolución conceptual de la evaluación educativa y de sus renovadas funciones sociales, la están situando en el punto central del quehacer didáctico, lo que se traduce en una función pedagógica basada en un mayor uso formativo de la evaluación incorporándola a los procesos de enseñanza- aprendizaje, en el sentido de ajustar o redireccionar las prácticas pedagógicas y apoyar el progreso de los aprendizajes del estudiante.

No se trataría de una tarea fácil el avanzar en la instalación de prácticas pedagógicas en el contexto universitario que permitan hacer realidad la evaluación en su dimensión formativa, y, por tanto, configurarla en espacio de retroalimentación para el aprendizaje del estudiantado. Desde una lógica de jerarquías, y desigualdades el desafío implica superar la noción de evaluación en tanto práctica patológica, y por tanto disfuncional respecto a los procesos que teóricamente pretende favorecer; selectiva en su afán por consagrar diferencias que en el discurso declara atender, reproductiva en términos de un escaso compromiso con la noción de aprendizaje como construcción activa de conocimiento; y por ende contradictoria, en el sentido de atrapada en un conjunto de usos éticamente cuestionables (Santos, 2003; Perrenoud,2008),

En la intención de consagrar la unidad indisoluble entre la enseñanza, el aprendizaje y la evaluación (Sadler, 2010), la función pedagógica de la misma emerge como la respuesta a lo planteado desde los modelos basados en competencia. Lograr que la función pedagógica, de análisis de los procesos, de diagnóstico, de comprensión, de diálogo, de retroalimentación y de participación decidida del estudiante (Jonsson, 2013; Orsmond et al., 2013) orienten los procesos formativos, implica superar el determinismo de la función social que la ancla a ser tenida como mecanismo de control y de comprobación exclusiva de los aprendizajes. Esto desafía a las universidades en general y a la formación de profesores en particular a diversificar los entornos de aprendizaje reconociendo las diferencias de los estudiantes y las limitaciones de nuestro sistema educativo. "Ello implica en la educación superior y especialmente en los docentes emprender cambios en la enseñanza para avanzar hacia prácticas donde el profesor sea un gestor, guía y facilitador del aprendizaje" (Gutiérrez, et al., 2020, p. 130), lo que desde la evaluación implica evaluación formativa y retroalimentación.

En el último tiempo se viene desarrollando investigación que pone de manifiesto distintas dimensiones de la evaluación formativa y la retroalimentación. Por una parte, los procesos de evaluación formativa y retroalimentación pueden ayudar a los estudiantes a tomar control de su propio aprendizaje, tornándose en aprendices autorregulados (Nicol y Macfarlane, 2007; Coll et al., 2012). Sin embargo, es preciso reconocer que la evaluación, constituye al mismo tiempo, el componente de los sistemas educativos menos permeable a los cambios, vale decir, en la práctica, la evaluación se suele mostrar abiertamente resistente a los esfuerzos de innovación de las prácticas pedagógicas. Se presenta como un proceso complejo de instalar en el contexto universitario, pues supone reconocer que la eficacia de esta retroalimentación implica requerimientos técnicos, y particularmente la credibilidad y capacidades del docente que la proporciona (Poulos y Majony, 2008; Price et al., 2010).

A la base de esta forma de entender la evaluación, está la centralidad del proceso en el estudiante, por tanto la evaluación debe ser capaz de promover el aprendizaje, favorecer la motivación y la autoevaluación (Andrade \& Valtcheva, 2009; Cauley \& McMillan, 2010), lo que para modelos academicistas y centradas en el conocimiento factual plantea el desafío de revisitar en profundidad el territorio de la evaluación formativa (Sadler, 2010; Dyan, 2006) haciendo frente al fuerte influjo que en los sistemas educativos universitarios juega la calificación y que terminan por condicionar la actuación del estudiante hacia aquello que se evalúa, y no necesariamente hacia el aprendizaje (Lizzio \& Wilson, 2008; Santos, 2003).

Para ello, se requiere una mirada más integradora de la evaluación en sus dimensiones sumativa y formativa, pues estos dos tipos de evaluación, las funciones que representan y las concepciones que esconden son complementarios y las diferencias deben ser superadas en la forma en que se utilizan y al servicio de qué usos se colocan (Dixson \& Worrell, 2016). No obstante, desde la perspectiva del estudiantado la evaluación universitaria se vive como un proceso esencialmente sumativo y, por tanto, de carácter social, existiendo menos evidencia productiva respecto a la forma en que estos conciben y valoran la evaluación formativa y sus beneficios. De ahí surge entonces el interés investigativo de comprender las principales paradojas que enfrenta la evaluación del aprendizaje en el contexto universitario de pedagogía. Contexto particularmente sensible porque el modelo de evaluación al que se enfrentan los futuros profesores por vía de sus formadores será muy probablemente replicado por ellos en su propio ejercicio docente vía apropiación por modelaje o vía aprendizajes a través del currículo oculto que través de la evaluación condiciona el aprendizaje y el comportamiento de los estudiantes. 


\section{METODOLOGÍA}

Este estudio se enmarca dentro de un enfoque de investigación cualitativa, de tipo hermenéutica y tiene la intención de discutir, desde la vivencia de estudiantes universitarios de pedagogía, las paradojas que muestra la evaluación del aprendizaje en su relación a los modelos basados en competencias y resultados de aprendizaje. A partir de un muestreo de tipo intencionado, se trabajó con 35 estudiantes de educación básica de la Universidad Católica del Maule quienes ofrecieron sus experiencias en torno al proceso de evaluación. De este grupo, 25 corresponden a mujeres y 10 varones, que cursan el tercer o cuarto año de formación. Los criterios para elegirles fueron: 1) Tener una experiencia formativa igual o superior a tres años (seis semestres); 2) ser estudiante de pedagogía básica; y 3) Aceptar participar de forma voluntaria, tras firma de consentimiento.

Se optó por un abordaje de tipo cualitativo, toda vez que la investigación pretende la comprensión subjetiva de los sujetos en relación a la evaluación (Vassilachis, 2006). El diseño utilizado fue de tipo transeccional no experimental con un nivel de profundidad exploratorio-descriptivo. El abordaje metodológico se desarrolló mediante el uso de una entrevista semiestructurada (Flick, 2007) a estudiante de pedagogía, la cual fue construida a partir del concepto de paradojas (Santos, 2003) y permitió indagar en torno a la evaluación del aprendizaje en el aula universitaria. Se dispuso el uso de esta aproximación metodológica, con guión de preguntas previamente validado, la que permitió acceder a datos descriptivos y a un abordaje interpretativo de los sujetos investigados.

El análisis de los datos se realizó mediante la Teoría Fundamentada. La primera etapa, de codificación abierta, consistió en el examen y fragmentación de la información recopilada para organizar los materiales en conceptos, ideas y categorías. Siguiendo los planteamientos de Corbin y Strauss (2008), los datos de las entrevistas fueron examinados y comparados, al amparo de una aproximación inductiva a la realidad, que permitió identificar las dimensiones y construir categorías pertinentes a la investigación. Para llevar a cabo la sistematización de la información, la Tabla 1 registra la frecuencia de paradojas presentes en la evaluación desde la perspectiva de estudiantes universitarios, lo que permite apreciar la densidad de la información, y en definitiva de los relatos:

Tabla 1: Frecuencia de paradojas presentes en la evaluación por dimensión y categorías

\begin{tabular}{|l|l|c|}
\hline Dimensión & Categorías & Frecuencia \\
\hline $\begin{array}{l}\text { Paradojas } \\
\text { de la } \\
\text { evaluación }\end{array}$ & Evaluación para aprender versus evaluación para aprobar & 30 \\
\cline { 2 - 3 } & Evaluación de proceso versus evaluación de resultados & 29 \\
\cline { 2 - 3 } & Evaluación para potenciar el pensamiento versus evaluación para reproducción & 25 \\
\cline { 2 - 3 } & Evaluación para mejorar procesos versus evaluación para repetir y conservar & 15 \\
\hline
\end{tabular}

\section{RESULTADOS}

Los resultados se presentan organizados en cuatro categorías: aprender versus aprobar; proceso versus resultados; pensamiento versus reproducción; y mejorar versus conservar. Cada categoría es acompañada de extractos de registros de habla.

\section{Evaluación para aprender versus evaluación para aprobar}

Este ámbito pone en tensión la finalidad de la evaluación vinculada al valor del conocimiento que las instituciones comunican a los estudiantes y el uso que se le termina otorgando. En relación a ello, la Tabla 2 registra las paradojas que los estudiantes universitarios declaran experimentar. En esta categoría, la paradoja que experimenta la evaluación se relaciona con la tensión entre constituirse en dispositivo promotor para el aprendizaje o mecanismo de control en función del cual se decide la trayectoria académica del estudiante. Específicamente, los estudiantes abogan por una evaluación formativa que sirva al aprendizaje en la medida que se hace efectiva la necesaria retroalimentación; sin embargo, la predominancia que tiene el valor de cambio o canje que representa la calificación para seguir avanzando en la trayectoria formativa termina por reducir el aprendizaje a su expresión de conocimiento y reproducción, con poco reconocimiento de la diversidad de estudiantes presente en el aula universitaria lo que se ve reflejado en la escasa variedad de instrumentos evaluativos que se utilizan. Se declaran conscientes de una evaluación que privilegia la selección, la cuantificación y la simplificación del proceso lo que redundaría en las escasas posibilidades de aprender. Ello generaría como consecuencia un estilo estudiantil centrado en la aprobación y que termina por adoptar un enfoque superficial de aprendizaje y, por tanto, instrumental en términos de "nadar o hundirse". 
Tabla 2: Paradoja sobre la finalidad de la evaluación

\begin{tabular}{|l|l|}
\hline Categorías de análisis & Extractos de registros de habla \\
\hline Evaluación para aprender versus & "Pocos docentes realizan el proceso de retroalimentación necesario posterior a \\
la evaluación para favorecer el aprendizaje, como la calificación ya está otorgada \\
al estudiante, se avanza en el contenido" (E3) \\
"Muchas veces el estudiante busca aprobar para el beneficio económico - social \\
más que por el beneficio propio de aprender realmente" (E35) \\
"En matemática es permanente la reprobación, ya que a la complejidad creciente \\
se suma una práctica donde no suele existir un monitoreo adecuado del proceso, \\
al contrario, prima la aclaración de dudas vagas, sugerencias pobres, lo que \\
reduce todo a nadar o hundirse, con el miedo a no ser capaz de salir adelante" \\
(E17) \\
"Los profesores no evalúan el proceso de enseñanza, sino más bien verificar el \\
resultado de un contenido, y así dar paso a que los alumnos solo memoricen para \\
lograr una buena calificación y aprobar la asignatura. Por lo mismo, no hay \\
instancias de autoevaluación o coevaluación" (E29) \\
"Muchas veces los profesores se limitan a una sola forma de evaluación y por \\
tanto, no atienden a la diversidad del aula" (E26)
\end{tabular}

Se confirma la presencia de una evaluación que sigue reproduciendo la creación de jerarquías de excelencia, un instrumento de homogeneización y selección social (Perrenoud, 2008) que no se correspondería con el compromiso que suponen los modelos basados en competencia en relación a enseñanza y evaluación. Los estudiantes entrevistados abogan por la necesidad de una evaluación al servicio del aprendizaje (Andrade y Valtcheva, 2009; Cauley y McMillan, 2010).

\section{Evaluación de proceso versus evaluación de resultados}

Este ámbito pone en discusión la intención de proceso o de resultados que suele caracterizar el ejercicio de la práctica evaluativa. En relación a ello, la Tabla 3 registra las paradojas que los estudiantes universitarios declaran experimentar. En esta categoría, la paradoja que experimenta la evaluación se relaciona con la tirantez entre su función social y pedagógica, según lo cual la práctica evaluativa en el contexto universitario si bien en el discurso proclama un compromiso con el proceso y el aprendizaje del estudiantado, termina por constituirse en una práctica anclada al resultado final, y al valor que atribuye a las calificaciones.

Tabla 3: Paradoja sobre el foco de la evaluación

\begin{tabular}{|l|l|}
\hline Categorías de análisis & Extractos de registros de habla \\
\hline $\begin{array}{l}\text { Evaluación de proceso } \\
\text { versus evaluación de } \\
\text { resultados }\end{array}$ & $\begin{array}{l}\text { "En las evaluaciones de ciencias o matemática, es frecuente que gran parte del curso se } \\
\text { equivo en el resultado de ejercicios, no así en el proceso, sin embargo, el o la docente } \\
\text { solo evalúa la solución final de cada estudiante. El error cuesta caro" (E2) } \\
\text { "La mayoría del tiempo estamos preocupados de "pasar el ramo" para así no atrasarnos, } \\
\text { sin enfocarnos en el aprendizaje significativo, "pasar" y luego olvidar lo aprendido. Muchas } \\
\text { veces nos cuestionamos que es lo que realmente sabemos ya que vamos avanzando sin } \\
\text { detenernos a pensar en lo que hemos aprendido o debemos reforzar" (E19) } \\
\text { "Muchas veces antes de realizar una evaluación, los estudiantes consideran el porcentaje } \\
\text { de esta, para verificar en muchas ocasiones si "les faltará nota" o si tienen que estudiar } \\
\text { mucho para poder rendir la evaluación y poder aprobar el ramo" (E17) } \\
\text { "En las evaluaciones de trabajo en grupo, siempre se distribuyen las preguntas a realizar } \\
\text { para optimizar el tiempo, dedicándose cada uno a su parte. Se sabe que no es lo ideal, pero } \\
\text { resulta" (E22) } \\
\text { "Aprobar es cuestión de vida o muerte, pues no se observa una preocupación de los } \\
\text { profesores por el aprendizaje, lo único que les preocupa es pasar el programa y seleccionar } \\
\text { en función de una nota. Para ellos la nota es sinónimo de aprendizaje" (E25) }\end{array}$ \\
\hline
\end{tabular}

La escasa atención al proceso, determina que el momento de la verdad se sitúa hacia el final de la enseñanza cuando la evaluación sumativa, a través de instrumentos de prueba certifica el nivel de logros alcanzados. En función de ello, la evaluación genera en el estudiantado la movilización de una serie de estrategias para adaptarse a los requerimientos de sus profesores. Entre ellas está la práctica de dividir el trabajo cuando este tiene un carácter grupal; calcular los porcentajes requeridos en las evaluaciones para decidir sobre los esfuerzos necesarios tendientes a asegurar aprobación; y asociar aprendizaje a aprobación y posterior olvido de lo supuestamente adquirido. Los estudiantes entrevistados advierten la escasa atención al proceso formativo por parte de sus profesores, lo que despierta en ellos incertidumbre respecto al aprendizaje que van 
logrando. Vale decir, no experimentan acciones de retroalimentación que genere algún tipo de aporte, sea este en términos instrumentales (mejorar calificaciones), de desarrollo (posibilidad para seguir aprendiendo), o motivacionales (inspiración para salir adelante). De hecho, la práctica de la evaluación se instrumentaliza en el uso negativo del error, lo que implica que "el error cuesta caro". Asimismo, reconocen en sus actuaciones estudiantiles la dispar consideración que otorgan a la reflexión sobre los aprendizajes efectivos que están logrando y que caen en la lógica de aprendizaje aprendido, aprobado y olvidado. Se confirma la presencia de una evaluación que no impacta en su función formativa (Ion y Cano, 2012; Coll, et al., 2012; Villardón, 2006), pues se traduce en una práctica disfuncional y patológica (Santos 2003) respecto a las oportunidades que ofrece al estudiantado para asegurar su aprendizaje. Ello impone retos al profesorado en el sentido de lograr avanzar hacia el ejercicio de acciones de retroalimentación (Evans, 2013; Poulos y Mahony, 2008; Price, et al., 2010)

\section{Evaluación para potenciar el pensamiento versus evaluación para reproducción}

Este ámbito pone en tensión la intención de la evaluación respecto al tipo de aprendizaje que favorece en el estudiantado. En relación a ello, la Tabla 4 registra las paradojas que los estudiantes universitarios declaran experimentar:

Tabla 4: Paradoja sobre el tipo de aprendizaje que favorece la evaluación

\begin{tabular}{|l|l|}
\hline Categorías de análisis & Extractos de registros de habla \\
\hline $\begin{array}{l}\text { Evaluación para } \\
\text { potenciar el } \\
\text { pensamiento versus } \\
\text { evaluación para } \\
\text { reproducción }\end{array}$ & $\begin{array}{l}\text { "Durante el proceso de enseñanza-aprendizaje de un ramo de historia, pudimos darnos } \\
\text { cuenta de que las clases solo enfatizaban contenido conceptual, dejando de lado el } \\
\text { desarrollo de otras habilidades. Asimismo, las evaluaciones correspondientes que se hacían } \\
\text { al finalizar el semestre, resultaban ser pruebas con preguntas cerradas y sin espacio para } \\
\text { la reflexión" (E3) } \\
\text { "En un ramo de ciencias, la profesora realizaba evaluaciones todas las clases, las cuales } \\
\text { consistían en memorizar el contenido de la clase anterior. Además, en todas las clases } \\
\text { hablaba de diferentes científicos y mencionaba que debíamos "memorizarlos" para la prueba } \\
\text { final" (E12) } \\
\text { "Es habitual responder pensando en lo que el académico(a) quiere que se conteste, en vez } \\
\text { de juzgar, analizar a los autores o contenidos asignados. De esta forma entra en nosotras } \\
\text { la presión, desde un comienzo por contestar bien, a consecuencia de frases en aula como } \\
\text { "Esto va entrar en la prueba" "no olviden esto que será pregunta de prueba", etc." (E18) } \\
\text { "Mayoritariamente, las evaluaciones a las cuales me he visto sometido no implican un } \\
\text { desafío que me permita desarrollar el espíritu crítico. En la práctica, veo -la gran mayoría } \\
\text { de las evaluaciones- como un proceso en el cual reproducimos" (E5) }\end{array}$ \\
\hline
\end{tabular}

En esta categoría, la paradoja que experimenta la evaluación se relaciona con el tipo de habilidades que en su instrumentación termina favoreciendo. Existiría un discurso categórico respecto a que la evaluación no constituiría un espacio para evidenciar el aprendizaje de saberes complejos o de habilidades de orden superior, sino más bien, seguiría atrapada en la verificación reproductiva de información de carácter conceptual, generando de esa manera una cultura que tiende a la simplificación del proceso formativo. Esta simplificación que asume la evaluación se ve ampliamente reflejada en un estudiantado que termina por adecuarse al estilo evaluador del profesor que enfrenta, el responder pensando en lo que cada profesor espera escuchar o leer; y en el predominio de la prueba de conocimiento y preguntas se selección múltiple como medio preferentes de evaluación. Asimismo, es notorio el proceso de condicionamiento que desde la evaluación el profesorado universitario realiza con los estudiantes para ajustar el actuar de este al interior del aula y enfrentar el proceso formativo: Así el estudiante asume que aprender y aprobar pasa por ser capaz de llegar a descubrir lo que el académico(a) quiere que se conteste.

Lo anterior pone de manifiesto varias implicancias para los actuales modelos formativos basados en competencias los que precisamente apuestan a innovar prácticas docentes ancladas al verbalismo y la reproducción, el control y la selección (Tejada y Ruiz, 2016; Jover y García, 2015; Juarros, 2006, Ion y Cano, 2012). Así como también a lograr la integración de saberes para contextos complejos de desempeño profesional (Chan, et al., 2017). Y probablemente, lo más preocupante sería que la supuesta centralidad en el estudiante y su aprendizaje que estos modelos postulan no estaría haciéndose realidad, pues desde la evaluación el control por parte del profesorado evidenciaría una fuente de resistencia (Beneitone, et al., 2007; Biggs, 2007; Coll, et al., 2012). Ello además tiene implicancias para una formación inicial docente que enfrenta desafíos de mejoramiento y que reconoce el rol estratégico que el profesorado tiene en el desarrollo de un sistema educativo de calidad. 


\section{Evaluación para mejorar procesos versus evaluación para repetir y conservar}

Este ámbito pone en discusión la intención de mejora que busca y logra la evaluación respecto a favorecer el desarrollo del pensamiento o la reproducción en la docencia. En relación a ello, la Tabla 5 registra las paradojas que los estudiantes universitarios declaran experimentar.

Tabla 5: Paradoja sobre la potencialidad de la evaluación para mejorar procesos

\begin{tabular}{|l|l|}
\hline \multicolumn{1}{|c|}{ Categorías de análisis } & \multicolumn{1}{c|}{ Extractos de registros de habla } \\
\hline $\begin{array}{l}\text { Evaluación para mejorar } \\
\text { procesos versus evaluación } \\
\text { para repetir y conservar }\end{array}$ & $\begin{array}{l}\text { "Son los profesores normalmente los que deciden los tipos de evaluaciones con las } \\
\text { cuales nos van a evaluar y los porcentajes, como a ellos más le acomode, sin tener } \\
\text { en cuenta las opiniones de sus estudiantes" (E27) } \\
\text { "Resultan muy predecibles las evaluaciones o la enseñanza de algunos profesores } \\
\text { que repiten, por dos o más años consecutivos una misma clase sin adecuarla a los } \\
\text { elementos propios de cada año o contexto, Aunque debiese servir para tomar } \\
\text { decisiones para mejorar el proceso de enseñanza, estas no se toman ya que no se } \\
\text { evidencian, la mayoría de las veces modificaciones o mejoras" (E14) } \\
\text { "Los profesores tienen la costumbre de nombrar o citar autores relevantes, sin } \\
\text { embargo, siempre se utilizan los mismos expertos, lo cual genera, que no exista una } \\
\text { actualización de la información y a su vez, no se aprecie una mirada más actual de } \\
\text { los contenidos que el profesor quiere enseñarnos" (E8) } \\
\text { "El docente en la mayoría de las ocasiones considera tener la verdad absoluta, sin } \\
\text { permitirle al estudiante una respuesta distinta a la esperada. Causando que los } \\
\text { conocimientos que entrega parezcan una "verdad única" no sujeta a críticas" (E30) }\end{array}$ \\
\hline
\end{tabular}

En esta categoría, la paradoja que experimenta la evaluación se inclina a la existencia de una práctica que se circunscribe a la noción de continuidad y control, lo que termina por desaprovechar la posibilidad que tiene como espacio para reflexionar, asumir nuevos desafíos y, en definitiva, convertirla en plataforma de mejora efectiva de los procesos. Así la evaluación se configura en una práctica que decide el docente, siendo él quien impone los criterios evaluativos. Aferrado a la posesión de la verdad que reflejan los saberes académicos, reproduce año a año los instrumentos evaluativos, el contenido lo comunica como verdad absoluta sin posibilidad de ser cuestionada por un estudiante al que no se reconoce en sus saberes. En consecuencia, se evalúa para conservar y no para mejorar o transitar a un aula dialógica que genere espacios de participación al estudiantado en su evaluación, o la reflexión sobre la misma.

Desde el punto de vista teórico, se constata que el docente universitario a través de la evaluación administra un poder que le faculta para imponer criterios, aplicar pruebas y decidir cuáles han de ser las pautas de corrección. En consecuencia, los procesos formativos mostrarían escaso alineamiento con los modelos basados en competencias, pues en la práctica se reflejan notorias dificultades para hacer efectivo un enfoque de aprendizaje centrado en el estudiante y sus desempeños, asegurando así una formación más pertinente (Dylan, 2006; Tejada y Ruiz, 2016; Sadler, 2010) y en sintonía con los modelos basados en competencias.

Los resultados del estudio son coincidentes con investigaciones en la materia, al constatar que la docencia universitaria está en el centro de la formación de las personas y es clave para ofrecer a los estudiantes las competencias necesarias en un mundo complejo e incierto (Jover y García, 2015; Villardón, 2006; Coll, et al., 2012; Struyven, et al., 2005). Sin embargo, la forma en que se estaría instrumentando el desarrollo de la evaluación termina por instalar ciertos usos y prácticas que necesitan ser visibilizadas y superadas (Orsmond, et al., 2013; Dixson y Worrell, 2016; Biggs, 2007) si lo que se pretende verdaderamente es innovar las prácticas pedagógicas con una formación profesional centrada en el estudiante y su aprendizaje.

La evaluación se vive como un proceso paradojal por parte de este grupo de estudiantes, dada la coexistencia de planteamientos dispares entre lo que declara como intención y lo que termina siendo la práctica pedagógica. Práctica que se ve atravesada con persistencia y profundidad por una serie de acciones que en torno a la evaluación la configuran como un espacio valorado en términos de aprobación/reprobación más que de verdadero aprendizaje y que, por tanto, se experimenta desde la presión por la aprobación y la sanción del error. En definitiva, esta práctica evaluativa reflejaría la distancia que muestra la docencia universitaria respecto de los actuales modelos basados en competencias y su intención de centralidad en el estudiante y su aprendizaje. La presencia de esta diversidad de lógicas de trabajo vigentes al interior de las instituciones terciarias (Parris y Saville, 2011), reflejan a nivel de evaluación del aprendizaje las dificultades para articular la selección y la formación, el reconocimiento y la negación de las desigualdades en pro de una formación pertinente (Perrenoud, 2018; Santos, 2003; Murphy, 2010; Lizzio y Wilson, 2008). 
Emerge como desafío y recomendación para la institución de educación superior el reconocimiento del estudiante en términos de su diversidad y necesidades, y por otra, perseverar en acciones tendientes al desarrollo académico de la docencia. Ello demanda una docencia que para el caso de formación de profesores no puede seguir replicando métodos y prácticas tradicionales de evaluación, pues con ello se termina por contradecir lo que a nivel de intenciones se pretende lograr.

Finalmente, las implicancias del estudio permiten sostener la demanda a una formación profesional donde la evaluación está llamada a constituir un dispositivo que favorezca las decisiones didácticas y pedagógicas, sentida por quienes viven sus consecuencias como una instancia de aprendizaje y no solo control. Ello supone revisión del estilo evaluador docente presente en las aulas universitarias, para asegurar el necesario alineamiento con los requerimientos de los modelos basados en competencia y los requerimientos de las actuales Políticas Públicas para Formación Inicial Docente.

\section{CONCLUSIONES}

De acuerdo a los resultados de este estudio, se pueden extraer las siguientes conclusiones. Primero, la evaluación para este grupo de estudiantes constituye una forma de actuar y pensar dirigida a la obtención del éxito reflejada en la aprobación de la calificación, en una tarea que implica "nadar o hundirse" y, por tanto, disociada de la finalidad del aprender.

Segundo, la evaluación se instrumentaliza en el aula universitaria bajo la lógica de la comprobación de un aprendizaje situado al final del proceso bajo criterios de selección y control que implican el uso punitivo del error.

Tercero, se observa la presencia de un estilo evaluador por parte del profesorado universitario que tiene implicancias en la práctica que se genera en las aulas que forman profesores. Para el estudiantado eso implica el esfuerzo por identificar rápidamente dicho estilo y responder a él dada la intención de asegurar el éxito y la aprobación. Desde el punto de vista formativo ello implica reconocer que la presencia de un determinado estilo evaluador, según los estudiantes de carácter reproductivo, tiene consecuencias en el tipo y profundidad de los aprendizajes alcanzados. Este estilo evaluador termina condicionando la respuesta del estudiante.

Cuarto, la forma en que se desarrolla la evaluación en aulas determina los roles de docente y estudiante. En la práctica, la evaluación la decide y resuelve el profesorado según sus criterios de verdad, sin generar espacios para la participación activa del estudiante en este ámbito. En consecuencia, el estudiante debe adaptarse al estilo evaluador, sin asumir la centralidad en el proceso que los modelos basados en competencias postulan.

Quinto, se configura una cultura de la evaluación que potencia unas formas de actuar y de pensar que la distancia de la noción de formación y la mantiene anclada a la de selección y control. Se muestra como un espacio didáctico reticente al cambio y la innovación.

Sexto, en aulas universitarias que preparan profesores, la forma en que se desarrolla y experimenta la evaluación tiene impactos más graves que en otras profesiones por el riesgo de terminar replicando estos aprendizajes y sus prácticas en el ejercicio profesional. De esa manera se ponen en peligro los desafíos propios de la formación por competencias y los de la formación inicial de profesores.

Finamente, resulta clave la reflexión sobre la evaluación para visualizar si está favoreciendo la adopción de los nuevos modelos y prácticas que se intenta innovar o constituye un espacio de resistencia a los cambios que requieren impulsar las instituciones de educación superior a través de la docencia.

\section{REFERENCIAS}

Andrade, H., y Valtcheva, A., Promoting learning and achievement through self-assessment, https://doi.org/10.1080/00405840802577544, Theory Into Practice, 48(1), 12-19 (2009)

Beneitone, P., Esquetini,C., y otros cuatro autores, Reflexiones y perspectivas de la Educación Superior en América Latina, Publicaciones de la Universidad de Deusto, Bilbao (2007)

Biggs, J., Teaching for quality learning of university, Open University Press, New York (2007)

Cauley, K.M., y McMillan, J.H., Formative assessment techniques to support student motivation and achievement, https://doi.org/10.1080/00098650903267784, The Clearing House: A Journal of Educational Strategies, Issues and Ideas, 83(1), 1-6 (2010)

Chan, C.K., Fong, E.T., Luk, L.Y., y Ho, R., A review of literature on challenges in the development and implementation of generic competencies in higher education curriculum, https://doi: 10.1016/j.ijedudev.2017.08.010, International Journal of Educational Development, 57(1), 1-10 (2017) 
Coll, C., Mauri, M., y Rochera, M.J., La práctica de evaluación como contexto para aprender a ser un aprendiz competente, Profesorado Revista de curriculum y formación del profesorado, 16(1), $49-59$ (2012)

Corbin, J., y Strauss, A., Basics of qualitative research: Techniques and procedures for developing grounded theory, CA: SAGE, Los Angeles (2008)

Dixson, D.D., y Worrell, F.C., Formative and summative assessment in the classroom, http://doi.org/ 10.1080/00405841.2016.1148989, Theory Into Practice, 55(2), 153-159 (2016)

Dylan. W., Formative assessment: getting the focus right, Educational Assessment, http://doi.org/10.1080/10627197.2006.9652993, Educational Assessment, 11(3), $283-289$ (2006)

Evans, C., Making sense of assessment feedback in higher education, https://doi.org/10.3102/0034654312474350, Review of Educational Research, 8(1), 70-120 (2013)

Flick, U., Introducción a la investigación cualitativa, $2^{\circ}$ ed., Morata, Madrid (2007)

Gutiérrez, X.D., Barría, C.M., y Tapia, C.P., Diseño universal para el aprendizaje de las matemáticas en la formación inicial del profesorado, http://dx.doi.org/10.4067/S0718-50062020000600129, Formación Universitaria, 13(6), 129-142 (2020)

Ion, G., y Cano, E., La formación del profesorado universitario para la implementación de la evaluación por competencias, https://doi.org/10.5944/educxx1.15.2.141, Educación XX1, 15(2), 249-270 (2012)

Jonsson, A., Facilitating productive use of feedback in higher education, https://doi.org/10.1177/1469787412467125, Active Learning in Higher Education, 14(1), 63-76 (2013)

Jover, G., y García, A., Relectura de la educación por competencias desde el pragmatismo de John Dewey, http://dx.doi.org/10.14201/eks20151613243, Education in the Knowledge Society, Salamanca, 16(1), 32-43 (2015)

Juarros, M.F., ¿Educación superior como derecho o como privilegio? Las políticas de admisión a la universidad en el contexto de los países de la región, Andamios, 3(5), 69-90 (2006).

Lizzio, A., y Wilson, K., Feedback on assessment: students' perceptions of quality and effectiveness,

https://doi.org/10.1080/02602930701292548, Assessment \& Evaluation in Higher Education, 33(3), $263-275$ (2008)

Murphy, C., Student perceptions of feedback: seeking a coherent flow, Practitioner Research in Higher Education, 4(1) 41-51 (2010)

Nicol, D., y Macfarlane, D., Formative assessment and self-regulated learning: a model and seven principles of good feedback practice, https://doi.org/10.1080/03075070600572090, Studies in Higher Education, 31(2), 199-218 (2007)

Orsmond, P., Maw, S., y otros tres autores, Moving feedback forward: theory to practice, https://doi.org/10.1080/02602938.2011.625472, Assessment \& Evaluation in Higher Education, 38(2), $240-252$ (2013)

Parris, M.A., y Saville, K., Piecing togetherthe puzzle of graduate employment: factors that shape the graduate work expectations of human resource management students, industry and, https://doi.org/10.5367/ihe.2011.0024, Higher Education, 25(1), 15-24 (2011)

Perrenoud, P., La evaluación de los alumnos. De la producción de la excelencia a la regulación de los aprendizajes. Entre dos lógicas, Colihue, Buenos Aires (2008)

Poulos, A., y Majony, M.J., Effectiveness of feedback: the students' perspective, https://doi.org/10.1080/02602930601127869, Assessment \& Evaluation in Higher Education, 33(2), 143-154 (2008)

Price, M., Handley, K., Millar, J., y O” Donovan, B., Feedback: all that effort, but what is the effect?, https://doi.org/10.1080/02602930903541007, Assessment \& Evaluation in Higher Education, 35(3), $277-289$ (2010)

Sadler, D.R., Beyond feedback: developing student capability in complex appraisal, https://doi.org/10.1080/02602930903541015, Assessment \& Evaluation in Higher Education, 35(5), 535-550 (2010)

Santos, M.A., Una flecha en la diana. La evaluación como aprendizaje, Narcea, S.A. Ediciones, Madrid (2003)

Struyven, K., Dochy, F., y Janssens, S., Students' perceptions about evaluation and assessment in higher education: a review, http://doi.org/10.1080/02602930500099102, Assessment and Evaluation in Higher Education, 30(4), 325-341 (2005)

Tejada, J., y Ruiz, C., Evaluación de competencias profesionales en educación superior: retos e implicaciones, http://doi.org/10.5944/educXX1.12175, Educación XXI, Madrid, 19 (1), 17-38 (2016)

Vasilachis, I., Estrategias de investigación cualitativa, Gedisa, Barcelona (2006)

Villardón, M.L., Evaluación del aprendizaje para promover el desarrollo de competencias, Educatio Siglo XXI, 24, 57- 76 (2006) 
